OPEN ACCESS

Edited by: Wen-Long $\mathrm{Hu}$

Kaohsiung Chang Gung Memorial

Hospital, Taiwan

Reviewed by:

Giustino Orlando,

University of Studies G.d'Annunzio

Chieti and Pescara, Italy

Pao-Yen Lin,

Kaohsiung Chang Gung Memorial

Hospital, Taiwan

*Correspondence:

Hui-fang Cong

hlichf196210.@sina.com

Specialty section:

This article was submitted to

Ethnopharmacology,

a section of the journal

Frontiers in Pharmacology

Received: 09 December 2021

Accepted: 31 January 2022

Published: 16 February 2022

Citation:

Gao Q, Shen L, Jiang B, Luan Y-f, Lin L-n, Meng F-C, Wang C-y and Cong H-f (2022) Salvia miltiorrhizaContaining Chinese Herbal Medicine

Combined With GnRH Agonist for

Postoperative Treatment of Endometriosis: A Systematic Review and meta-Analysis.

Front. Pharmacol. 13:831850. doi: 10.3389/fphar.2022.831850

\section{Salvia miltiorrhiza-Containing Chinese Herbal Medicine Combined With GnRH Agonist for Postoperative Treatment of Endometriosis: A Systematic Review and meta-Analysis}

\author{
Qiang Gao ${ }^{1}$, Lei Shen ${ }^{2}$, Bei Jiang ${ }^{1}$, Yi-feng Luan ${ }^{3}$, Li-na Lin ${ }^{1}$, Fan-ci Meng ${ }^{1}$, Chao-ying Wang ${ }^{1}$ \\ and Hui-fang Cong ${ }^{1,3 *}$
}

\footnotetext{
${ }^{1}$ Graduate School, Heilongjiang University of Traditional Chinese Medicine, Harbin, China, ${ }^{2}$ Department of Traditional Chinese Medicine, Aerospace Center Hospital, Beijing, China, ${ }^{3}$ The First Department of Gynecology, The Second Affiliated Hospital of Heilongjiang University of Traditional Chinese Medicine, Harbin, China
}

Background: Endometriosis is an estrogen-dependent gynecological inflammatory condition that may lead to infertility and recurrent pelvic pain. The purpose of this research was to determine the efficacy and safety of Salvia miltiorrhiza-containing Chinese herbal medicine (CHM) combined with gonadotropin-releasing hormone agonist $(\mathrm{GnRH}-\mathrm{a})$ for postoperative endometriosis management.

Methods:Eight databases were systematically searched before October 2021, including PubMed, Embase, Cochrane Library, Scopus, Web of Sceince, CNKI, VIP, and Wanfang. Finally, all randomized controlled studies comparing Salvia miltiorrhiza-containing $\mathrm{CHM}$ paired with $\mathrm{GnRH}$-a to $\mathrm{GnRH}$-a alone for postoperative endometriosis management were included.

Results: A total of 10 trials involving 836 patients were reported and analyzed. Compared with the control group, the Salvia miltiorrhiza-containing $\mathrm{CHM}$ combined with $\mathrm{GnRH}-\mathrm{a}$ group showed significant superiority in decreasing endometriosis recurrence (risk ratio $[R R]=0.26$; 95\% confidence intervals $[\mathrm{Cl}]: 0.16-0.41$ ) and increasing the pregnancy rate $([\mathrm{RR}]=1.96 ; 95 \% \mathrm{Cl}: 1.58-2.44)$. Similarly, the effect of the Salvia miltiorrhiza-containing $\mathrm{CHM}$ combined with $\mathrm{GnRH}-\mathrm{a}$ on $\mathrm{CA}-125$ serum levels was positive (standardized mean difference $[S M D]=-0.79 ; 95 \% \mathrm{Cl}:-1.11$ to -0.47$)$. Furthermore, this group showed a significant reduction in adverse effects.

Conclusion: The results indicate that Salvia miltiorrhiza-containing CHM may be a viable choice for postoperative endometriosis therapy, with the potential to enhance pregnancy while decreasing recurrence and adverse effects.

Keywords: Salvia miltiorrhiza, GnRH-a, Chinese herbal medicine, endometriosis, systematic review, meta-analysis 


\section{INTRODUCTION}

Endometriosis is a gynecological disease characterized by the presence of endometrial epithelium and stroma outside the uterine cavity, which causes chronic pelvic pain, dysmenorrhea, deep dyspareunia, dysuria and infertility (Patzkowsky, 2021). Endometriosis affects between 5 and $10 \%$ of reproductive-age women worldwide. The true incidence of endometriosis may be higher due to factors such as undiagnosed and clinical misdiagnosis (Zondervan, et al., 2020; Taylor et al., 2021). Current therapy options mostly involve pharmacological and surgical treatments, and it is well known that laparoscopic surgery is regarded as the gold standard for endometriosis treatment (Sindan et al., 2021). Unfortunately, over 50\% of women undergoing surgery require further surgery within 5 years (Saraswat et al., 2018; Saunders and Horne, 2021). GnRH-a is frequently used after surgery to eliminate microscopic lesions and prevent recurrence of endometriosis. It inhibits follicle development and ovulation by lowering FSH and LH secretion, which decreases the synthesis of estradiol and progesterone to suppress the progression of the lesion and treat endometriosis-related pain (Della Corte et al., 2020). Nonetheless, the long-term use of GnRH-a causes adverse effects such as vasomotor symptoms, insomnia, and bone density loss (Sauerbrun-Cutler and Alvero, 2019). Although add-back treatment is commonly used to alleviate side effects, its efficacy remains inadequate (Donnez and Dolmans, 2021).
Chinese herbal medicine (CHM) has long been used to treat various gynecological disorders, including endometriosis. The herb Salvia miltiorrhiza has been officially listed in Chinese Pharmacopoeia for treatment of menstrual disorder and blood circulation diseases and prevention of inflammation (Kuang et al., 2005). In addition, the use of Salvia miltiorrhiza in combination with Gui Zhi Fu Ling Wan formulation $\left(\right.$ Gynoclear $^{\mathrm{TM}}$ ) is considered to reduce the severity and duration of aperiodic pelvic pain, dysmenorrhea, dyspareunia, and other endometriosis symptoms (Armour et al., 2021). Currently, known salvianolic compounds that have primarily pharmacological effects include tanshinone, salvianolic, rosmarinic, caffeic, protocatechuic and danshensu acids, which not only inhibit platelet aggregation and fibrosis, but also have anti-inflammatory, anti-oxidant effects, anti-cancer, and other pharmacological effects (Matkowski et al., 2008; Bostanci et al., 2021; Meresman et al., 2021). Tanshinone IIA, a pharmacologically active extract of Salvia miltiorrhiza, has been shown to control the renin-angiotensin system and diminish mechanical hyperalgesia in endometriosis pain (Chen and Gong, 2020). Furthermore, it inhibits the proliferation, migration, and invasion control of endometrial stromal cells, preventing lesions' evolution (Luo et al., 2020).

Cancer antigen 125 (CA-125) is a glycoprotein, which is a wellestablished tumour marker of the ovarian epithelial cells. At present, CA-125 is considered to be a potential marker of endometriosis and has been widely tested in the clinical diagnosis of endometriosis. Although CA-125 has a relatively low sensitivity and specificity, its high level is related to the stage and clinical type of endometriosis

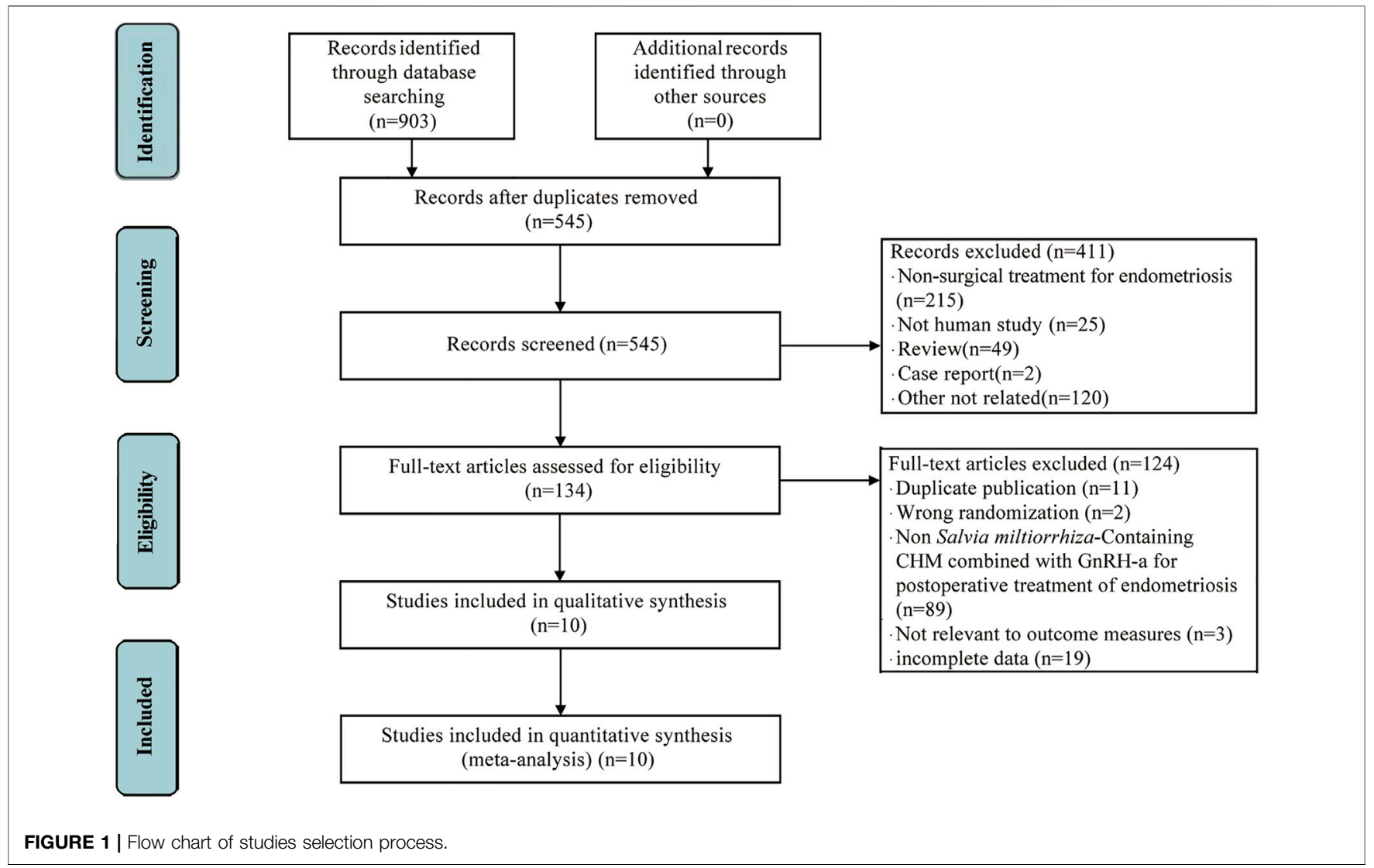


TABLE 1 | Characteristics of included studies.

\begin{tabular}{|c|c|c|c|c|c|c|c|}
\hline Study & $\begin{array}{l}\text { Sample size } \\
\text { (TG/CG) }\end{array}$ & Age(years) & Intervention group & Control group & Duration & $\begin{array}{l}\text { Follow-up } \\
\text { duration }\end{array}$ & Outcomes \\
\hline Chen (2019) & $100(50 / 50)$ & $\begin{array}{c}\text { TG: } \\
30.73 \pm 4.93 \\
\text { CG: } \\
30.90 \pm 4.75\end{array}$ & $\begin{array}{l}\text { Salvia-containing } \\
\text { CHM + Leuprolide } \\
\text { Acetate }\end{array}$ & Leuprolide Acetate & $\begin{array}{l}\text { CHM: } 21 \text { days * } 3 \text { courses } \\
\text { Leuprolide Acetate: Once } \\
\text { every } 28 \text { days }{ }^{*} 3 \text { courses }\end{array}$ & 12 months & (1) \\
\hline Du et al. (2018) & $60(30 / 30)$ & $\begin{array}{l}\text { TG: } 38.0 \pm 1.5 \\
\text { CG: } 37.1 \pm 1.5\end{array}$ & $\begin{array}{l}\text { Salvia-containing } \\
\mathrm{CHM}+\text { Goserelin } \\
\text { Acetate }\end{array}$ & Goserelin Acetate & $\begin{array}{l}\text { CHM: } 3 \text { weeks * } 6 \text { courses } \\
\text { Goserelin Acetate: Once every } \\
4 \text { weeks }{ }^{*} 6 \text { courses }\end{array}$ & 24 months & (1) (2) (3) (4) \\
\hline $\mathrm{Hu}(2016)$ & $92(46 / 46)$ & $\begin{array}{l}\text { TG: } 34.1 \pm 4.7 \\
\text { CG: } 33.9 \pm 4.9\end{array}$ & $\begin{array}{l}\text { Salvia-containing } \\
\mathrm{CHM}+\text { Triptorelin }\end{array}$ & Triptorelin & $\begin{array}{l}\text { CHM: } 4 \text { weeks * } 6 \text { courses } \\
\text { Triptorelin: Once every } \\
4 \text { weeks * } 6 \text { courses }\end{array}$ & 24 months & (1) (2) \\
\hline $\begin{array}{l}\text { Hu and Kuang } \\
\text { (2018) }\end{array}$ & $90(45 / 45)$ & $\begin{array}{l}\text { TG: } 30.9 \pm 5.5 \\
\text { CG: } 30.2 \pm 6.1\end{array}$ & $\begin{array}{l}\text { Salvia-containing } \\
\mathrm{CHM}+\text { Triptorelin } \\
\text { Acetate }\end{array}$ & Triptorelin Acetate & $\begin{array}{l}\text { CHM: } 14 \text { days }{ }^{*} 6 \text { courses } \\
\text { Triptorelin Acetate: Once every } \\
28 \text { days * } 6 \text { courses }\end{array}$ & 6 months & (2) (3) (4) \\
\hline $\begin{array}{l}\text { Lei and } \\
\text { Gao(2019) }\end{array}$ & $104(52 / 52)$ & $\begin{array}{c}\text { TG: } \\
28.71 \pm 5.06 \\
\text { CG: } \\
29.86 \pm 4.83\end{array}$ & $\begin{array}{l}\text { Salvia-containing } \\
\text { CHM + Leuprolide + } \\
\text { Estradiol Valerate }\end{array}$ & $\begin{array}{l}\text { Leuprolide + } \\
\text { Estradiol Valerate }\end{array}$ & $\begin{array}{l}\text { CHM: } 28 \text { days * } 6 \text { courses } \\
\text { Leuprolide: Once every } \\
28 \text { days }{ }^{*} 6 \text { courses }\end{array}$ & 36 months & (1) (2) (3) (4) \\
\hline Liu et al. (2021) & $100(52 / 48)$ & $\begin{array}{c}\text { TG: } \\
30.24 \pm 4.30 \\
\text { CG: } \\
29.74 \pm 4.12\end{array}$ & $\begin{array}{l}\text { Salvia-containing } \\
\text { CHM + Leuprolide } \\
\text { Acetate }\end{array}$ & Leuprolide Acetate & $\begin{array}{l}\text { CHM: } 12 \text { weeks } \\
\text { Leuprolide Acetate: Once } \\
\text { every } 4 \text { weeks }{ }^{*} 3 \text { courses }\end{array}$ & 12 months & (1) (2) (4) \\
\hline Wu (2016) & $60(30 / 30)$ & $\begin{array}{c}\text { TG: } \\
35.6 \pm 2.62 \\
\text { CG: } \\
36.6 \pm 2.49\end{array}$ & $\begin{array}{l}\text { Salvia-containing } \\
\text { CHM + Triptorelin } \\
\text { Acetate }\end{array}$ & Triptorelin Acetate & $\begin{array}{l}\text { CHM: } 21 \text { days * } 3 \text { courses } \\
\text { Triptorelin Acetate: Once every } \\
28 \text { days * } 3 \text { courses }\end{array}$ & 6 months & (2) (3) \\
\hline Zhang (2020) & $98(49 / 49)$ & $\begin{array}{c}\text { TG: } \\
35.02 \pm 2.13 \\
\text { CG: } \\
34.56 \pm 2.67\end{array}$ & $\begin{array}{l}\text { Salvia-containing } \\
\text { CHM + Triptorelin } \\
\text { Acetate }\end{array}$ & Triptorelin Acetate & $\begin{array}{l}\text { CHM: } 3 \text { weeks * } 6 \text { courses } \\
\text { Triptorelin Acetate: Once every } \\
4 \text { weeks * } 6 \text { courses }\end{array}$ & 12 months & (1) (2) (3) (4) \\
\hline $\begin{array}{l}\text { Zhang and } \\
\text { Wang (2019) }\end{array}$ & $60(30 / 30)$ & $\begin{array}{c}\text { TG: } \\
31.63 \pm 5.12 \\
\text { CG: } \\
30.17 \pm 4.47\end{array}$ & $\begin{array}{l}\text { Salvia-containing } \\
\text { CHM + Leuprolide } \\
\text { Acetate }\end{array}$ & Leuprolide Acetate & $\begin{array}{l}\text { CHM: } 21 \text { days * } 3 \text { courses } \\
\text { Leuprolide Acetate: Every } \\
28 \text { days }{ }^{*} 3 \text { courses }\end{array}$ & 12 months & (1) (2) (3) (4) \\
\hline $\begin{array}{l}\text { Zhang and Zhao } \\
\text { (2017) }\end{array}$ & $72(36 / 36)$ & $\begin{array}{l}\text { TG: } 30.7 \pm 4.6 \\
\text { CG: } 31.9 \pm 5.0\end{array}$ & $\begin{array}{l}\text { Salvia-containing } \\
\mathrm{CHM}+\text { Leuprolide } \\
\text { Acetate }\end{array}$ & Leuprolide Acetate & $\begin{array}{l}\text { CHM: } 21 \text { days * } 3 \text { courses } \\
\text { Leuprolide Acetate: Once } \\
\text { every } 28 \text { days }{ }^{*} 3 \text { courses }\end{array}$ & 12 months & (2) (4) \\
\hline
\end{tabular}

CHM = chinese herbal medicine; (1)pregnancy rate; (2)recurrence rate; (3) CA-125; (4)adverse events.

and is more sensitive to stages III and IV of endometriosis (KimberTrojnar et al., 2021). It is recommended that concentrations of CA125 be measured during the middle of the menstrual cycle and during the menstrual period. In particular, positive results for CA125 in the middle of the menstrual cycle suggest a very high risk of endometriosis (Oliveira et al., 2017).

No previous systematic reviews or meta-analyses have been conducted to investigate the efficacy of Salvia miltiorrhizacontaining $\mathrm{CHM}$ in postoperative endometriosis patients. Therefore, this study aimed to evaluate the effect of Salvia miltiorrhiza-containing $\mathrm{CHM}$ combined with $\mathrm{GnRH}-\mathrm{a}$ to reduce the risk of recurrence and adverse effects, and promote pregnancy in postoperative endometriosis patients.

\section{MATERIALS AND METHODS}

\section{Data Sources and Search Strategy}

The search was conducted utilizing the following electronic databases to October 2021: PubMed, Embase, Cochrane Library,
Scopus, Web of Sceince, China National Knowledge Infrastructure (CNKI), Journal Integration Platform (VIP) and Wanfang. Furthermore, to minimize publication bias, we manually evaluated the references of all selected studies and searched for related papers such as letters, research reports, research papers, conference proceedings, and abstracts. Medical subheadings ( $\mathrm{MeSH}$ ) words combined with free words were used for retrieval in the English library, and the search terms (endometriosis OR endometrioses OR endometrioma OR endometriomas) AND (Chinese Traditional Medicine OR Chinese herbal medicine) AND (GnRH agonist OR Gonadotropin-releasing hormone agonist OR GnRH-a) were used as keywords.

\section{Selection Criteria Types of Studies}

Included studies were human randomized controlled trials (RCTs) published in English or Chinese only. Non-RCTs, in vitro studies, and animal studies were removed. Reviews, case reports, abstracts, and repeated publications were also excluded. 


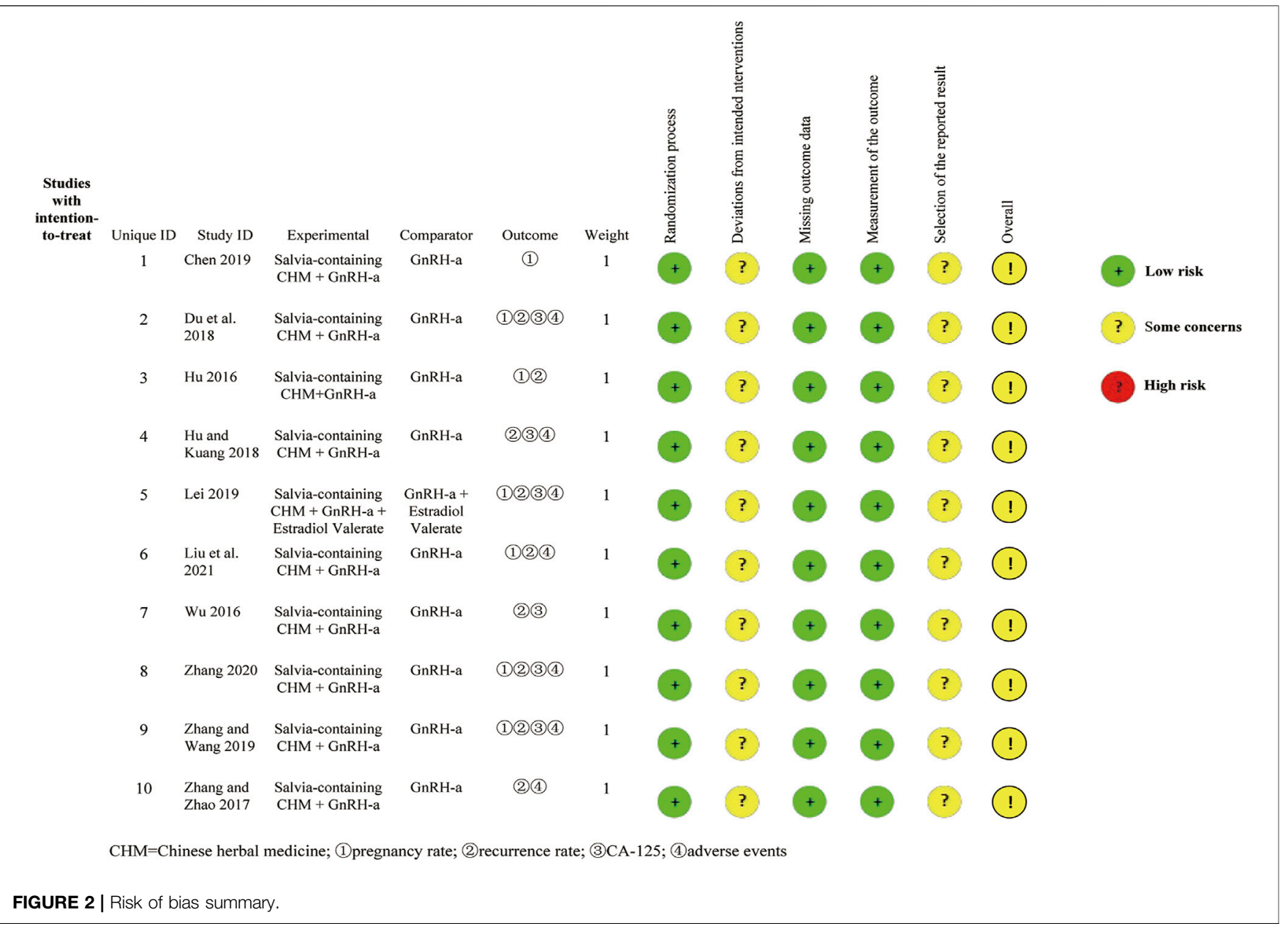

\section{Types of Patients}

Patients had a clear diagnosis of endometriosis, which was confirmed by pathological diagnosis of laparoscopic surgery or conservative surgery by laparotomy. Additionally, following surgery, all patients in the intervention and control groups were given GnRH-a therapy.

\section{Types of Interventions}

Patients in the intervention groups received Salvia miltiorrhizacontaining CHM combined with GnRH-a as postoperative medical treatment, while patients in the control groups received GnRH-a alone therapy after surgery. Salvia miltiorrhiza-containing CHM was available as capsules, tablets, pills, and decoctions. Studies using Chinese nonherbal medicinal therapies such as acupuncture, external enema, cupping, or point application were excluded.

\section{Types of Outcome Measures}

The primary outcomes were endometriosis recurrence rates. Secondary outcomes included pregnancy rate, CA-125 level in peripheral blood, and adverse events such as gastrointestinal reaction, vaginal bleeding, hot flashes and abnormal liver function.

\section{Data Extration}

Two authors independently selected the articles based on inclusion and exclusion criteria. The following information was extracted using a standardized data collection form: first author, publication year, study design, sample sizes, intervention details, outcomes, and follow-up duration. When two authors disagreed, a third author joined the discussion to achieve an agreement.

\section{Quality Assessment}

Two independent reviewers assessed the methodological quality of each trial using the Cochrane Handbook for Systematic Reviews of Interventions, which included items such as randomization process, deviations from intended interventions, missing outcome data, measurement of the outcome, selection of the reported result, and overall bias (Higgins et al., 2019).

\section{Statistical Analysis}

The data analysis was performed with STATA software (version 16.0, Stata Corporation, College Station, TX, United States). Dichotomous variables were shown as risk ratio (RR) with 95\% confidence intervals (CI), and continuous variables were presented as standardized mean difference (SMD) with 95\%. 


\section{Study}

ID
Events, Events, \%

$\mathrm{RR}(95 \% \mathrm{Cl}) \quad$ experiment control Weight

\begin{tabular}{|c|c|c|c|c|}
\hline Du et al. (2018) & $0.20(0.05,0.84)$ & $2 / 30$ & $10 / 30$ & 13.14 \\
\hline Hu (2016) & $0.33(0.10,1.09)$ & $3 / 25$ & $8 / 22$ & 11.18 \\
\hline Hu and Kuang (2018) & $0.30(0.09,1.02)$ & $3 / 45$ & $10 / 45$ & 13.14 \\
\hline Lei (2019) & $0.32(0.11,0.93)$ & $4 / 51$ & $12 / 49$ & 16.08 \\
\hline Liu et al. (2021) & $0.21(0.05,0.90)$ & $2 / 52$ & 9/48 & 12.30 \\
\hline Zhang (2020) & $0.22(0.05,0.98)$ & $2 / 49$ & 9/49 & 11.82 \\
\hline Zhang and Wang (2019) & $0.22(0.05,0.94)$ & $2 / 30$ & $9 / 30$ & 11.82 \\
\hline Zhang and Zhao (2017) & $0.25(0.06,1.10)$ & $2 / 36$ & $8 / 36$ & 10.51 \\
\hline Overall $(I-$ squared $=0.0 \%, p=0.999)$ & $0.26(0.16,0.41)$ & $20 / 318$ & $75 / 309$ & 100.00 \\
\hline
\end{tabular}

FIGURE 3 | Forest plot showing comparision of recurrence rate comparing Salvia-containing CHM combined with GnRH-a to GnRH-a alone treatment.

Homogeneity across trails was evaluated using the $I^{2}$ statistics, and $I^{2}>50 \%$ was assumed to have high heterogeneity. We applied a fixed-effect model to assess treatment effects. A $p$-value $<0.05$ was considered statistically significant.

\section{RESULTS}

The screening process is shown in Figure 1, 903 articles were selected by searching Pubmed $(n=11)$, Embase $(n=14)$, the Cochrane library $(\mathrm{n}=9)$, Scopus $(\mathrm{n}=2)$, Web of Sceince $(n=15)$, CNKI $(n=191)$, VIP $(n=241)$, and the Wangfang database $(n=$ 420). A total of 358 articles were removed after deleting the duplicates. 411 articles were excluded after scanning the titles and abstracts. Following the full texts 134 studies reviewed, only 10 studies met the criteria were finally included in this systematic review and meta analysis.

\section{Study Characteristics}

All included studies were conducted in China and published in Chinese between 2016 and 2021. Table 1 shows the main characteristics of the trails, which consisted of sample size, age, duration, and outcomes. 10 studies recruited a total of 836 postoperative patients with endometriosis, including 420 patients in Salvia miltiorrhiza-containing CHM combined with $\mathrm{GnRH}-\mathrm{a}$ group and 416 patients in $\mathrm{GnRH}-\mathrm{a}$ alone group.

\section{Quality Assessment}

Figure 2 shows the methodological quality assessment of included studies. All studies were described as randomized, of which 7 studies used random tables. There were no statistically significant differences in baseline between the intervention and control groups across all enrolled studies. None of the included studies reported blind intervention for patients. There was no information that the intervention deviated from the intended intervention due to the experimental context. Outcome data was obtained for nearly all randomized groups of subjects. Although all studies were unclear on the blinding of outcome assessment, patients with endometriosis have objective evaluation indexes for recurrence, pregnancy and serum CA-125 level, and it was difficult to affect the outcomes evaluation. No information mentioned that the results analysed in accordance with published pre-specified analysis plan. Consistent outcome measures and data analysis methods were used for all included studies. Overall bias showed some concerns.

\section{Recurrence Rate}

As shown in Figure 3, a fixed effect model was used due to no obvious heterogeneity observed $\left(I^{2}=0 \%, p=0.999\right)$. The result $(\mathrm{RR}=0.26$; 95\%CI: 0.16-0.41) indicated that Salvia miltiorrhizacontaining CHM plus GnRH-a was superior to GnRH-a alone in decreasing the recurrence rate. 


\section{A Pragnancy rate \\ Study}

ID
Events, Events, \%

$\mathrm{RR}(95 \% \mathrm{Cl}) \quad$ experiment control Weight

\begin{tabular}{|c|c|c|c|c|}
\hline Chen (2019) & $3.00(1.40,6.42)$ & $21 / 50$ & $7 / 50$ & 9.40 \\
\hline Du et al. (2018) & $1.89(1.01,3.55)$ & $17 / 30$ & $9 / 30$ & 12.08 \\
\hline $\mathrm{Hu}(2016)$ & $1.69(1.01,2.84)$ & $20 / 29$ & $11 / 27$ & 15.30 \\
\hline Lei (2019) & $1.62(1.01,2.62)$ & $27 / 51$ & $16 / 49$ & 21.91 \\
\hline Liu et al. (2021) & $1.75(1.18,2.59)$ & $36 / 52$ & $19 / 48$ & 26.53 \\
\hline Zhang (2020) & $3.67(1.09,12.34)$ & $11 / 49$ & $3 / 49$ & 4.03 \\
\hline Zhang and Wang (2019) & $2.13(1.09,4.16)$ & $17 / 30$ & $8 / 30$ & 10.74 \\
\hline Overall $(\mathrm{I}$-squared $=0.0 \%, p=0.738)$ & $1.96(1.58,2.44)$ & $149 / 291$ & $73 / 283$ & 100.00 \\
\hline
\end{tabular}

\section{B Serum level of CA-125.}

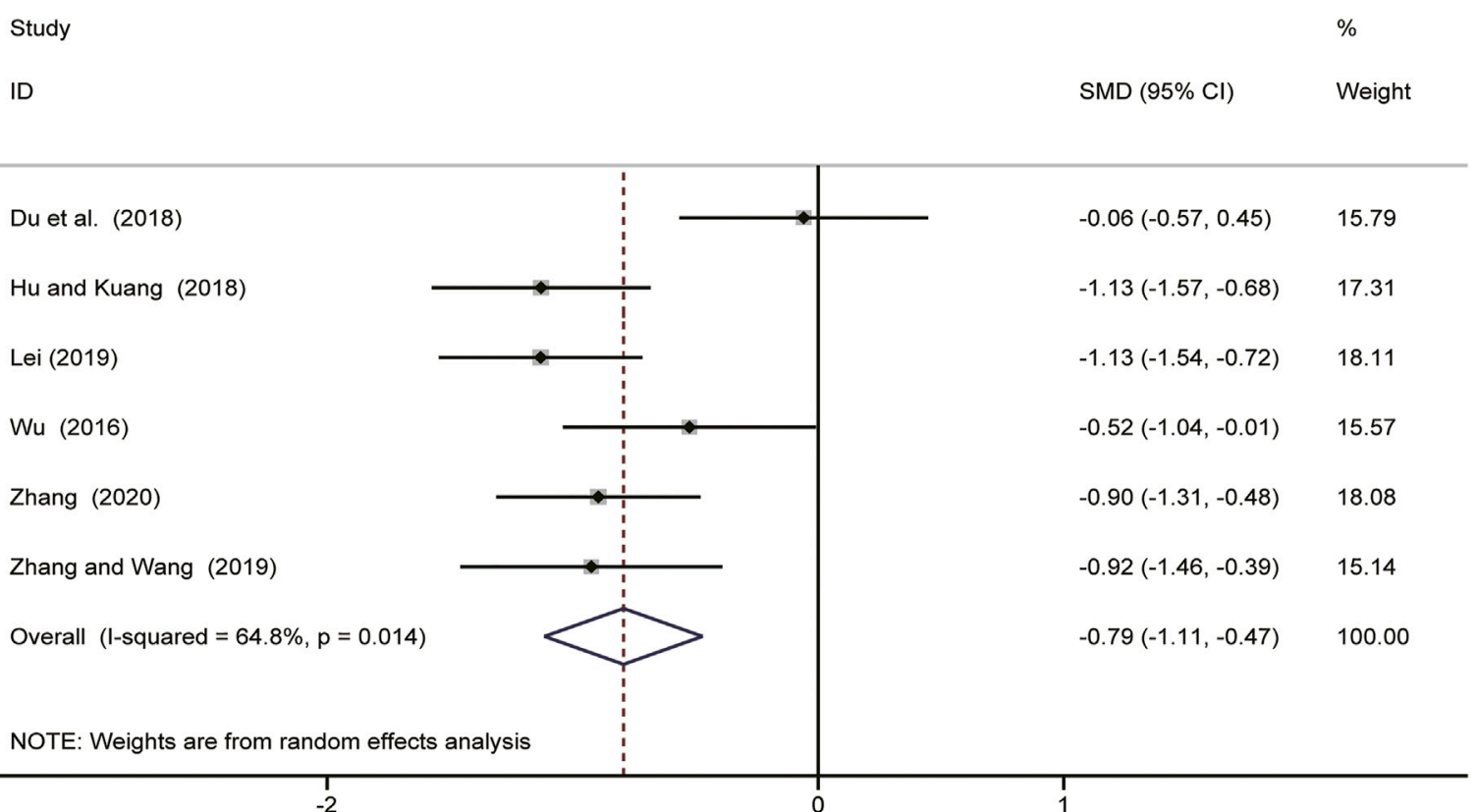

FIGURE 4 | Forest plots showing comparision of pregnangcy rate (A) and serum level of CA-125 (B) comparing Salvia-containing CHM combined with GnRH-a to $\mathrm{GnRH}-\mathrm{a}$ alone treatment.

Pregnancy Rate and Serum Level of CA-125 As shown in Figure 4A, we applied a fixed effect model because no obvious heterogeneity was observed $\left(I^{2}=0 \%, p=0.738\right)$. The result $(\mathrm{RR}=1.96 ; 95 \% \mathrm{CI}: 1.58-2.44)$ showed that postoperative Salvia miltiorrhiza-containing CHM combined with $\mathrm{GnRH}-\mathrm{a}$ therapy significantly increased the endometriosis pregnancy rate. Six studies compared the variation in serum CA-125 level between intervention and control groups. As depicted in Figure 4B, metaanalysis using a random model suggested that postoperatie Salvia miltiorrhiza-containing CHM combined with GnRH-a treatment remarkably reduced serum level of CA-125 (SMD $=-0.79,95 \% \mathrm{CI}$ : -1.11 to -0.47$)$ compared to GnRH-a alone. 
Gastroitestinal reaction

Study

ID
Events, Events, \%

$\mathrm{RR}(95 \% \mathrm{Cl}) \quad$ experiment control Weight

Du et al. (2018)

Hu and Kuang (2018)

Zhang (2020)

Overall (I-squared $=0.0 \%, p=0.443$ )

.01

$0.67(0.12,3.71) \quad 2 / 30 \quad 3 / 30 \quad 37.50$

$2.00(0.19,21.28) \quad 2 / 45 \quad 1 / 45 \quad 12.50$

$0.25(0.03,2.16) \quad 1 / 49 \quad 4 / 49 \quad 50.00$

$0.63(0.21,1.86) \quad 5 / 124 \quad 8 / 124 \quad 100.00$

30

Vaginal bleeding

Study

ID
Events, Events, \%

$\mathrm{RR}(95 \% \mathrm{Cl})$ experiment control Weight

Lei (2019)

Liu et al. (2021)

Zhang (2020)

Overall (I-squared $=0.0 \%, p=0.876$ )

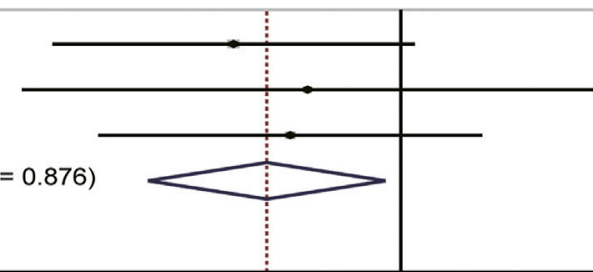

$0.25(0.06,1.12) \quad 2 / 52 \quad 8 / 52 \quad 53.05$

$0.46(0.04,4.93) \quad 1 / 52 \quad 2 / 48 \quad 13.79$

$0.40(0.08,1.96) \quad 2 / 49 \quad 5 / 49 \quad 33.16$

$0.33(0.12,0.88) \quad 5 / 153 \quad 15 / 149 \quad 100.00$

Hot flashes

Study

ID

Du et al. (2018)

Lei (2019)

Liu et al. (2021)

Wu (2016)

Zhang and Zhao (2017)

Overall (I-squared $=0.0 \%, p=0.879$ )

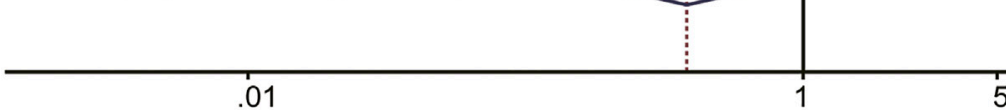

Events, Events, \%

$\mathrm{RR}(95 \% \mathrm{Cl})$ experiment control Weight

$\begin{array}{llll}0.17(0.02,1.30) & 1 / 30 & 6 / 30 & 19.23\end{array}$

$0.50(0.10,2.61) \quad 2 / 52 \quad 4 / 52 \quad 12.82$

$0.55(0.14,2.19) \quad 3 / 52 \quad 5 / 48 \quad 16.67$

$0.42(0.17,1.04) \quad 5 / 30 \quad 12 / 30 \quad 38.46$

$\begin{array}{llll}0.25(0.03,2.13) & 1 / 36 & 4 / 36 & 12.82\end{array}$

$0.38(0.20,0.71) \quad 12 / 200 \quad 31 / 196 \quad 100.00$

Abnormal liver function Study

ID

Events, Events, \% $\operatorname{RR}(95 \% \mathrm{Cl})$ experiment control Weight

\begin{tabular}{|c|c|c|c|c|}
\hline Du et al. (2018) & $0.33(0.04,3.03)$ & $1 / 30$ & $3 / 30$ & 37.50 \\
\hline Zhang (2020) & $0.20(0.02,1.65)$ & $1 / 49$ & $5 / 49$ & 62.50 \\
\hline Overall $(I-$ squared $=0.0 \%, p=0.742)$ & $0.25(0.05,1.14)$ & $2 / 79$ & $8 / 79$ & 100.00 \\
\hline
\end{tabular}

FIGURE 5 | Forest plots showing comparision of adverse events comparing Salvia-containing $\mathrm{CHM}$ combined with $\mathrm{GnRH}$-a to $\mathrm{GnRH}$-a alone treatment.

\section{Adverse Events}

Adverse events including gastrointestinal reactions, vaginal bleeding, hot flashes and abnormal liver function, were reported in 7 studies. As shown in Figure 5, meta-analysis indicated that the incidence of irregular vaginal bleeding $(\mathrm{RR}=$
0.33 ; $95 \% \mathrm{CI}: 0.12$ to $\left.0.88 ; I^{2}=0 \%, p=0.876\right)$ and hot flashes $(\mathrm{RR}$ $=0.38 ; 95 \%$ CI: 0.20 to $0.71 ; \mathrm{I}^{2}=0 \%, p=0.879$ ) were lower in the Salvia miltiorrhiza-containing CHM combined with GnRH-a group than in the GnRH-a alone group. However, there were no significant differences on the risk of gastrointestinal reaction 


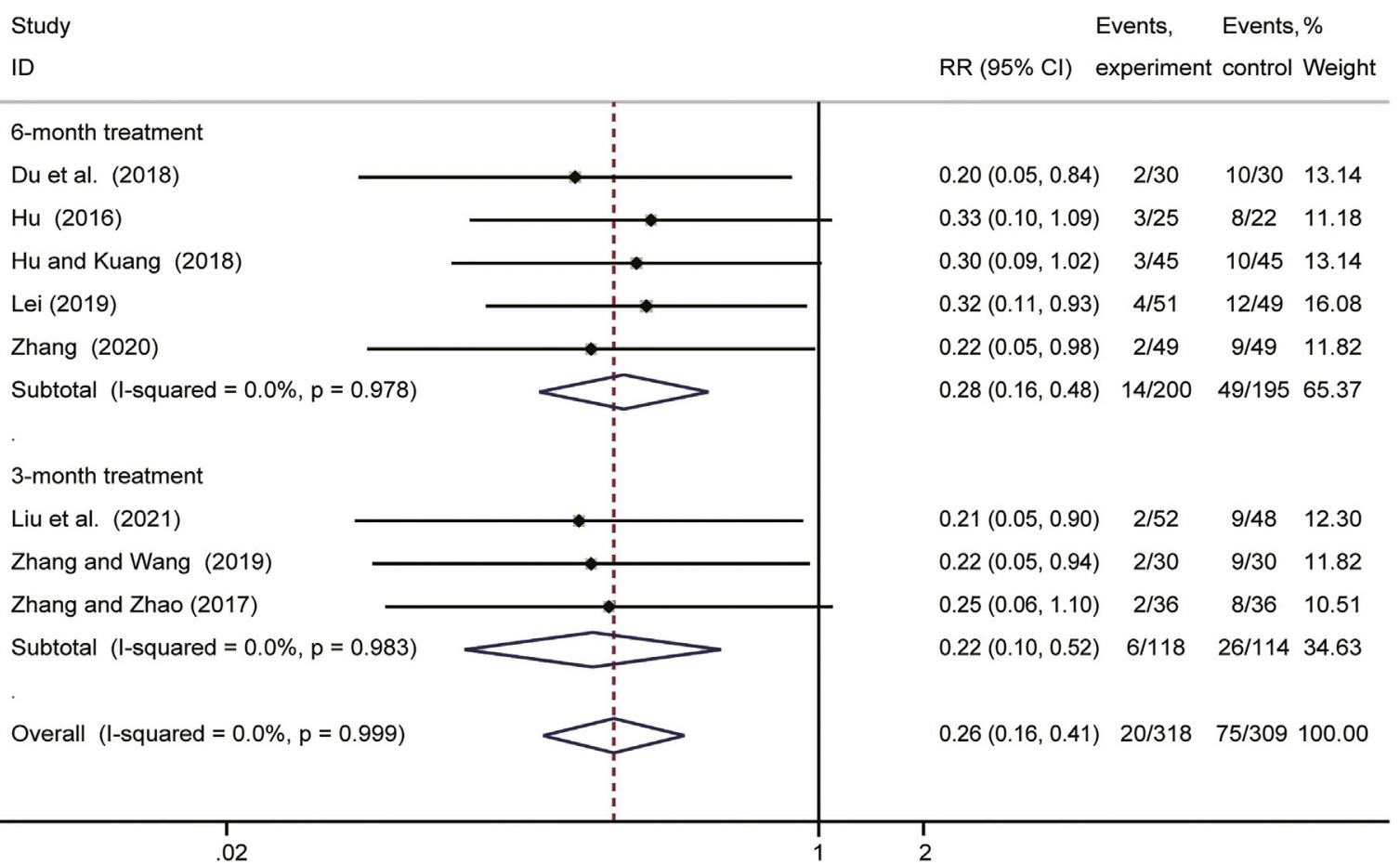

FIGURE 6 | Forest plot of the effect of different durations of postoperative Salvia-containing $\mathrm{CHM}$ combined with GnRH-a treatment in preventing endometriosis recurrence.

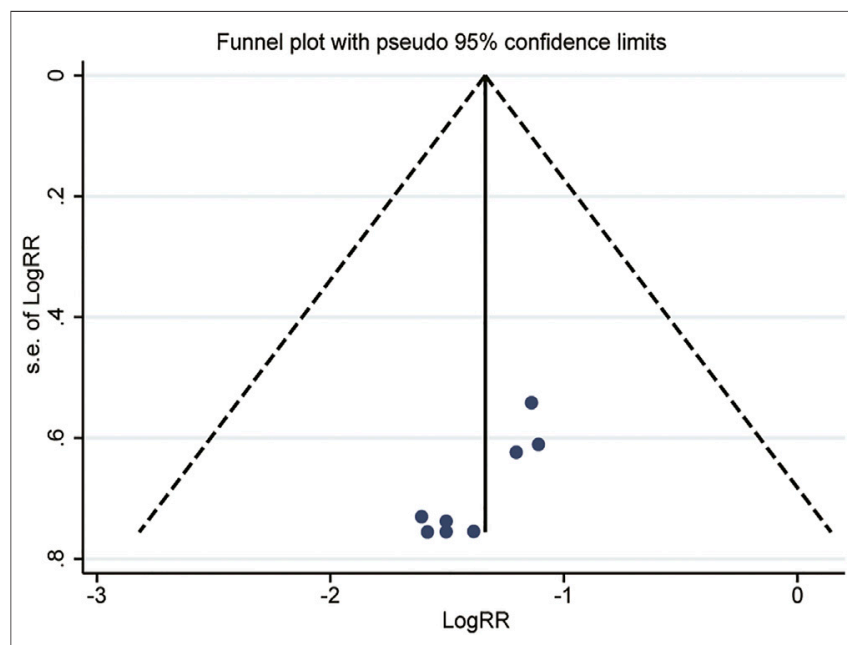

FIGURE 7 | Funnel plot of the recurrence rate.

$\left(\mathrm{RR}=0.63 ; 95 \% \mathrm{CI}: 0.21\right.$ to $\left.1.86 ; I^{2}=0 \%, p=0.443\right)$ and abnormal liver function ( $\mathrm{RR}=0.25 ; 95 \% \mathrm{CI}$ : 0.05 to $1.14 ; I^{2}=$ $0 \%, p=0.742$ ).

\section{Subgroup Analyses and Sensitivity Analysis}

According to the duration of therapy, we conducted the subgroup analysis on the recurrence rate in postoperative patients with endometriosis. The results revealed that the recurrence rate in patients received 6 months postoperative
Salvia miltiorrhiza-containing CHM combined with GnRH-a treatment ( $\mathrm{RR}=0.28$; 95\% CI: $0.16-0.48$ ) was much lower than those received 3 months treatment $(\mathrm{RR}=0.22 ; 95 \% \mathrm{CI}$ : 0.10-0.52) (Figure 6). The results of this meta-analysis can be considered stable since no significant changes were noted in the leave-one-out sensitivity analysis.

\section{Publication Bias}

We used a series of approaches to investigate potential publication bias. Figure 7 presented that the funnel plot was generally symmetrical visually, and the result of Harbord's test $(p=0.143)$ also confirmed it, which showed that publication bias was not obvious.

\section{Grade Evaluation of Evidence Quality}

According to the GRADE standard (Guyatt et al., 2008), GRADE profiler 3. 6 was used to evaluate the evidence quality of each outcome. Outcome indexes were classified into four grades of high quality, medium quality, low quality and extremely low quality according to five aspects of research limitation, inconsistency, inaccuracy, indirectness and other biases. The Evidence Profifile with quality assessment and Summary of Findings were reported in Table 2.

\section{DISCUSSION}

Endometriosis is a common disease in women of reproductive age. Because of the mild symptoms at the beginning of the disease, 
TABLE 2 | GRADE rating of the quality of each outcome.

\begin{tabular}{|c|c|c|c|c|c|}
\hline \multicolumn{6}{|c|}{ Anticipated absolute effects ${ }^{*}(95 \% \mathrm{Cl})$} \\
\hline \multirow[b]{2}{*}{ Outcomes } & \multirow{2}{*}{$\frac{\text { Risk with }}{\text { [comparison] }}$} & \multirow{2}{*}{$\begin{array}{c}\text { Risk with } \\
\text { [intervention] }\end{array}$} & \multirow{2}{*}{$\begin{array}{c}\text { Relative effect } \\
(95 \% \mathrm{Cl})\end{array}$} & \multirow{2}{*}{$\begin{array}{c}\begin{array}{c}\text { № of } \\
\text { participants }\end{array} \\
\text { (studies) }\end{array}$} & \multirow{2}{*}{$\begin{array}{c}\begin{array}{c}\text { Certainty of } \\
\text { the evidence }\end{array} \\
\text { (GRADE) }\end{array}$} \\
\hline & & & & & \\
\hline Recurrence rate & 243 per 1,000 & 63 per 1,000 (39-100) & RR 0.26(0.16-0.41) & 627 (8 RCTs) & $\begin{array}{c}\square \square \square \square \\
\text { High }\end{array}$ \\
\hline Pregnancy rate & 258 per 1,000 & 506 per 1,000 (408-629) & RR $1.96(1.58-2.44)$ & $574(7 \mathrm{RCTs})$ & $\begin{array}{c}\square \square \square \hat{x} \\
\text { Moderate }\end{array}$ \\
\hline serum level of CA-125 & - & SMD 0.79 SD lower (1.11 lower to 0.47 lower) & - & 472 (6 RCTs) & $\begin{array}{c}\square \square \square \hat{x} \\
\text { Moderate }\end{array}$ \\
\hline Gastrointestinal reactions & 65 per 1,000 & 41 per $1,000<(14-120)$ & RR $0.63(0.21-1.86)$ & 248 (3 RCTs) & $\begin{array}{c}\square \square \hat{x} \hat{x} \\
\text { Low }\end{array}$ \\
\hline Vaginal bleeding & 101 per 1,000 & 33 per 1,000 (12-89) & RR 0.33(0.12-0.88) & $302<(3$ RCTs $)$ & $\begin{array}{c}\square \square \square \hat{x} \\
\text { Moderate }\end{array}$ \\
\hline Hot flashes & 158 per 1,000 & 60 per $1,000(32-112)$ & RR $0.38(0.20-0.71)$ & 396 (5 RCTs) & $\begin{array}{c}\square \square \square \hat{x} \\
\text { Moderate }\end{array}$ \\
\hline Abnormal liver function & 101 per 1,000 & 25 per $1,000(5-115)$ & RR 0.25 (0.05-1.14) & 158(2 RCTs) & $\begin{array}{c}\square \square \hat{x} \hat{x} \\
\text { Low }\end{array}$ \\
\hline Recurrence rate(3-month treatment) & 228 per 1,000 & 50 per $1,000(23-119)$ & RR $0.22(0.10-0.52)$ & 232 (3 RCTs) & $\begin{array}{c}\square \square \square \hat{x} \\
\text { Moderate }\end{array}$ \\
\hline Recurrence rate(6-month treatment) & 251 per 1,000 & 70 per $1,000(40-121)$ & RR $0.28(0.16-0.48)$ & 395 (5 RCTs) & $\begin{array}{c}\square \square \square \hat{x} \\
\text { Moderate }\end{array}$ \\
\hline
\end{tabular}

${ }^{*}$ The risk in the intervention group (and its 95\% confidence interval) is based on the assumed risk in the comparison group and the relative effect of the intervention (and its $95 \%$ Cl). Cl: confidence interval; RR: risk ratio; SMD: standardised mean difference.

patients do not seek immediate medical attention until the endometriosis has developed to a severe degree, increasing treatment difficulties. Some studies in recent years have comfirmed the clinical effects of Salvia miltiorrhiza-containing CHM for angina, myocardial infarction, hepatitis, and dysmenorrhea, as well as its low toxic side effects (Wu et al., 2012; Liao et al., 2019). Still, there is no systematic evidence to show whether Salvia miltiorrhiza-containing CHM is suitable for endometriosis. As far as we know, this is the first systematic review and meta-analysis of RCTs on the efficacy of Salvia miltiorrhiza-containing CHM in the treatment of endometriosis.

The meta-analysis's main results revealed that, compared to GnRH-a therapy alone, Salvia miltiorrhiza-containing CHM + GnRH-a might reduce the recurrence rate of postoperative endometriosis patients. In addition, using Salvia miltiorrhizacontaining $\mathrm{CHM}$ as an adjuvant treatment to GnRH-a significantly increased pregnancy rates while decreasing peripheral blood CA-125 levels and side events. We systematically evaluated the results of 10 RCTs comprising 836 patients (420 in Salvia miltiorrhiza-containing CHM combined with GnRH-a group and 416 in the GnRH-a alone group).

Due to the high recurrence rate of endometriosis after surgery, inhibition of endogenous estrogen production is very important for the treatment of endometriosis (Bedaiwy et al., 2017). The combination of surgery and post-surgical medical treatment with GnRH-a is the most commonly used therapy for mediate and severe endometriosis, because $\mathrm{GnRH}-\mathrm{a}$ has been proven to be effective in removing microscopic lesions and suppressing the secretion of FSH, LH and estradiol (Donnez et al., 2002). The CA125 is usually described as a clinical marker for the diagnosis of endometriosis (Kimber-Trojnar et al., 2021). In the current meta-analysis, Salvia miltiorrhiza-containing CHM combined with GnRH-a significantly decreased the serum level of CA125 in postoperative patients with endometriosis. Moreover, the preclinical study has proved its effect of treating endometriosis by significantly reducing rat serum CA-125 levels and IL-18 in peritoneal fluid, and increasing the level of IL-13 in peritoneal fluid (Zhou et al., 2012).

However, the pharmacological mechanism of GnRH-a in the treatment of endometriosis is down-regulation of the pituitary and suppression of gonadotropin release to sustain the hypoestrogenic state, which causes mimic postmenopausal symptoms included irregular vaginal bleeding, hot flashes, headache (Rahmawati et al., 2019; Dai et al., 2021). Although hormone replacement "add-back" therapy is frequently prescribed as an adjunct therapy to GnRH-a to prevent menopausal side effects, long-term use of GnRH-a may lead to increasing the risk of osteoporosis (Alshehre et al., 2020). Given the above factors, the safety and effectiveness of using GnRH-a beyond 6 months are still controversial, which undoubtedly limit the clinical efficacy of GnRH-a (Bedaiwy and Casper, 2006; Hornstein, 2017). Therefore, it is necessary to seek complementary and alternative therapies to reduce the side effects of GnRH-a in the treatment of endometriosis. According to the results of this meta-analysis, Salvia miltiorrhiza-containing CHM combined with GnRH-a reduced the risk of vaginal bleeding and hot flashes caused by GnRH-a and did not significantly increase the incidence of gastrointestinal reactions and abnormal liver function. Furthermore, when taken the duration of Salvia miltiorrhiza-containing CHM treatment into account, we found that 6 months treatment with Salvia miltiorrhizacontaining $\mathrm{CHM}$ could better reduce the recurrence rate of 
endometriosis. These results indicated that the combination of Salvia miltiorrhiza-containing CHM and GnRH-a could improve the therapeutic effects. We believe that these benefits of Salvia miltiorrhiza-containing CHM make it a good candidate for the treatment of endometriosis.

There are several limitations in this study. First, some enrolled RCTs did not explicitly report a randomized approach, with only seven studies reported using random number tables and the remaining three mentioned randomization without supplying further details. None of the included studies reported implementation of a blinding method and using a placebo in the control group. These methodological flaws might generate bias, so that our results should be explained very carefully. Second, most studies lacked sample size evaluation, which leads to the low accuracy of most included small sample size studies. Third, the forms of Salvia miltiorrhiza-containing CHM were not unified, which might cause potential bias. Fourth, the missing stages of endometriosis reported in most trials might affect the pooling outcome for recurrence of endometriosis. Finally, all the included studies did not provide the approval results of the ethics committee, so we should pay attention to ethical issues and protect patients' reasonable rights.

In conclusion, the findings of this meta-analysis indicated that Salvia miltiorrhiza-containing CHM might be used as a supplemental therapy for postoperative endometriosis treatment. Salvia miltiorrhiza-containing CHM appears to be a potential medication for improving clinical effectiveness and lowering GnRH-a side effects. However, due to the low quality of most of the included studies, further large-scale and highquality, rigorous RCTs will be required in the future to confirm these results.

\section{REFERENCES}

Alshehre, S. M., Duffy, S., Jones, G., Ledger, W. L., and Metwally, M. (2020). A Prospective, single-centre, Single-Arm, Open Label Study of the Long Term Use of a Gonadotropin Releasing Hormone Agonist (Triptorelin SR, $11.25 \mathrm{mg}$ ) in Combination with Tibolone Add-Back Therapy in the Management of Chronic Cyclical Pelvic Pain. Reprod. Biol. Endocrinol. 18 (1), 28. doi:10.1186/s12958020-00586-z

Armour, M., Al-Dabbas, M. A., Ee, C., Smith, C. A., Ussher, J., Arentz, S., et al. (2021). The Effectiveness of a Modified Gui Zhi Fu Ling Wan Formulation $\left(\right.$ Gynoclear $^{\mathrm{TM}}$ ) for the Treatment of Endometriosis: a Study Protocol for a Placebo-Controlled, Double-Blind, Randomised Controlled Trial. Trials 22 (1), 299. doi:10.1186/s13063-021-05265-x

Bedaiwy, M. A., Allaire, C., and Alfaraj, S. (2017). Long-term Medical Management of Endometriosis with Dienogest and with a Gonadotropin-Releasing Hormone Agonist and Add-Back Hormone Therapy. Fertil. Steril 107 (3), 537-548. doi:10.1016/j.fertnstert.2016.12.024

Bedaiwy, M. A., and Casper, R. F. (2006). Treatment with Leuprolide Acetate and Hormonal Add-Back for up to 10 Years in Stage IV Endometriosis Patients with Chronic Pelvic Pain. Fertil. Steril 86 (1), 220-222. doi:10.1016/j.fertnstert.2005. 12.030

Bostanci, M. T., Bulbul, A. S., Celik, I. S., Kocabas, Y. Z., Burhan, H., Bayat, R., et al. (2022). Investigation of Antibacterial, Antifungal, Antibiofilm, Antioxidant and Anticancer Properties of Methanol Extracts of Salvia Marashica İlçim, Celep \& Doğan and Salvia Caespitosa Montbret \& Aucher Ex Benth Plants with Medicinal Importance. Chemosphere 288, 132602. doi:10.1016/j. chemosphere.2021.132602

\section{DATA AVAILABILITY STATEMENT}

The original contributions presented in the study are included in the article/Supplementary Material, further inquiries can be directed to the corresponding authors.

\section{AUTHOR CONTRIBUTIONS}

Hf-C contributed to the conception. QG and LS undertook curation. QG, LS, and BJ performed the statistical analysis. QG, Yf-L, and Ln-L wrote the original draft. QG, LS, BJ, Fc$\mathrm{M}$, and $\mathrm{Cy}-\mathrm{W}$ wrote the sections of manuscript.

\section{FUNDING}

This paper was supported by Heilongjiang University of Traditional Chinese Medicine in 2021.

\section{ACKNOWLEDGMENTS}

We thank professor Hf-C for her guidance on this meta-analysis methodology.

\section{SUPPLEMENTARY MATERIAL}

The Supplementary Material for this article can be found online at: https://www.frontiersin.org/articles/10.3389/fphar.2022.831850/ full\#supplementary-material

Chen, S. Y. (2019). "Effect of Nullation and Deflation of Kidney and GnRH-A on Postoperative Pregnancy of Endometriosis," (Fuzhou: Fujian University of Traditional Chinese Medicine), 3-12. M Sc. Thesis.

Chen, Z. Z., and Gong, X. (2020). Tanshinone IIA Contributes to the Pathogenesis of Endometriosis via Renin Angiotensin System by Regulating the Dorsal Root Ganglion Axon Sprouting. Life Sci. 240, 117085. doi:10.1016/j.lfs.2019.117085

Dai, Y., Shi, B., Huang, X., Duan, J., Qiu, Y., Ha, C., et al. (2021). Cost-effectiveness Analysis of Dienogest Compared with Gonadotropin-Releasing Hormone Agonist after Conservative Surgery for Endometriosis in China. Clin. Ther. 43 (8), 1276-e1. doi:10.1016/j.clinthera.2021.07.002

Della Corte, L., Barra, F., Mercorio, A., Evangelisti, G., Rapisarda, A. M. C., Ferrero, S., et al. (2020). Tolerability Considerations for Gonadotropin-Releasing Hormone Analogues for Endometriosis. Expert Opin. Drug Metab. Toxicol. 16 (9), 759-768. doi:10.1080/17425255.2020.1789591

Donnez, J., Chantraine, F., and Nisolle, M. (2002). The Efficacy of Medical and Surgical Treatment of Endometriosis-Associated Infertility: Arguments in Favour of a Medico-Surgical Aproach. Hum. Reprod. Update 8 (1), 89-94. doi:10.1093/humupd/8.1.89

Donnez, J., and Dolmans, M. M. (2021). GnRH Antagonists with or without AddBack Therapy: A New Alternative in the Management of Endometriosis? Int. J. Mol. Sci. 22 (21), 11342. doi:10.3390/ijms222111342

Du, Q. M., Guo, H. L., and Feng, H. F. (2018). Observation on the Clinical Efficacy of GnRh-A Combined with Huayu Xiaozheng Recipe on the Prevention of Conservative Postoperative Recurrence of Ovarian Endometriotic Cyst. J. Traditional Chin. Med. Res. 31 (08), 26-28. doi:10.3969/j.issn.1001-6910. 2018.08.11

Guyatt, G. H., Oxman, A. D., Vist, G. E., Kunz, R., Falck-Ytter, Y., Alonso-Coello, P., et al. (2008). GRADE: An Emerging Consensus on Rating Quality of 
Evidence and Strength of Recommendations. BMJ. 336 (7650), 924-926. doi:10. 1136/bmj.39489.470347.AD

Higgins, J., Thomas, J., Chandler, J., Miranda CTianjing, L., Matthew, P., et al. (2019). Cochrane Handbook for Systematic Reviews of Interventions Version 6.0 [Z]. Chichester, UK: John Wiley \& Sons.

Hornstein, M. D. (2017). An Oral GnRH Antagonist for Endometriosis - A New Drug for an Old Disease. N. Engl. J. Med. 377 (1), 81-83. doi:10.1056/ NEJMe1706000

Hu, F. L. (2016). Observation on the Curative Effect of Integrated Traditional Chinese and Western Medicine in the Treatment of 46 Cases of Endometriosis. New Chin. Med. 48 (03), 147-149. doi:10.13457/j.cnki.jncm.2016.03.058

Hu, Y. Y., and Kuang, H. Y. (2018). Clinical study of dan'e fukang soft extracts combined with triptorelin in treatment of ovarian chocolate cyst. Mod. Med. Clin. 33 (09), 2331-2335. doi:10.7501/j.issn.1674-5515.2018.09.038

Kimber-Trojnar, Ż., Pilszyk, A., Niebrzydowska, M., Pilszyk, Z., Ruszała, M., and Leszczyńska-Gorzelak, B. (2021). The Potential of Non-invasive Biomarkers for Early Diagnosis of Asymptomatic Patients with Endometriosis. J. Clin. Med. 10 (13), 2762. doi:10.3390/jcm10132762

Kuang, L., and Zhang, K.Chinese Pharmacopoeial Commission (2005). Pharmacopoeia of the People's Republic of China 2005. Beijing: People's Medical Publishing House.

Lei, J., and Gao, X. (2019). Effect of Modified Bushen Quyu Decoction on Inoculation Function for Patients with Endometriosis after Laparoscopic Surgery. Sichuan Traditional Chin. Med. 37 (12), 171-174.

Liao, W., Ma, X., Li, J., Li, X., Guo, Z., Zhou, S., et al. (2019). A Review of the Mechanism of Action of Dantonic ${ }^{\circledR}$ for the Treatment of Chronic Stable Angina. Biomed. Pharmacother. 109, 690-700. doi:10.1016/j.biopha.2018.10.013

Liu, Y., Feng, C. H., and Xue, J. (2021). Effects of Danhuang Quyu Capsules and Leuprolide Acetate Sustained Release Microspheres for Injection on Serum Inflammatory Factors and Sex Hormones in Patients with Endometriosis and Infertility after Laparoscopy. Hebei Traditional Chin. Med. 43 (01), 103-106. doi:10.3969/j.issn.1002-2619.2021.01.026

Luo, M., Cai, X., Yan, D., Liu, X., and Guo, S. W. (2020). Sodium Tanshinone IIA Sulfonate Restrains Fibrogenesis through Induction of Senescence in Mice with Induced Deep Endometriosis. Reprod. Biomed. Online 41 (3), 373-384. doi:10. 1016/j.rbmo.2020.04.006

Matkowski, A., Zielińska, S., Oszmiański, J., and Lamer-Zarawska, E. (2008). Antioxidant Activity of Extracts from Leaves and Roots of Salvia Miltiorrhiza Bunge, S. Przewalskii Maxim., and S. Verticillata L. Bioresour. Technol. 99 (16), 7892-7896. doi:10.1016/j.biortech.2008.02.013

Meresman, G. F., Götte, M., and Laschke, M. W. (2021). Plants as Source of New Therapies for Endometriosis: a Review of Preclinical and Clinical Studies. Hum. Reprod. Update 27 (2), 367-392. doi:10.1093/humupd/dmaa039

Oliveira, M. A. P., Raymundo, T. S., Soares, L. C., Pereira, T. R. D., and Demôro, A. V. E. (2017). How to Use CA-125 More Effectively in the Diagnosis of Deep Endometriosis. Biomed. Res. Int. 2017, 9857196. doi:10.1155/2017/9857196

Patzkowsky, K. (2021). Rethinking Endometriosis and Pelvic Pain. J. Clin. Invest. 131 (20), e154876. doi:10.1172/JCI154876

Rahmawati, E., Yang, W. V., Lei, Y. P., Maurya, P. K., Chen, H. W., and Tzeng, C. R. (2019). Decreased Level of Neurotrophic Factor Neuritin 1 in Women with Ovarian Endometriosis after Receiving Gonadotropin-Releasing Hormone Agonist Treatment. Int. J. Mol. Sci. 20 (18), 4352. doi:10.3390/ijms20184352

Saraswat, L., Ayansina, D., Cooper, K. G., Bhattacharya, S., Horne, A. W., and Bhattacharya, S. (2018). Impact of Endometriosis on Risk of Further
Gynaecological Surgery and Cancer: a National Cohort Study. BJOG 125 (1), 64-72. doi:10.1111/1471-0528.14793

Sauerbrun-Cutler, M. T., and Alvero, R. (2019). Short- and Long-Term Impact of Gonadotropin-Releasing Hormone Analogue Treatment on Bone Loss and Fracture. Fertil. Steril 112 (5), 799-803. doi:10.1016/j.fertnstert.2019.09.037

Saunders, P. T. K., and Horne, A. W. (2021). Endometriosis: Etiology, Pathobiology, and Therapeutic Prospects. Cell 184 (11), 2807-2824. doi:10. 1016/j.cell.2021.04.041

Sindan, N., Bhandari, A., Sindan, N., Kc, R., Xia, E., and Lin, Y. (2021). Clinical Factors Influencing the Pregnancy Outcome after Laparoscopic Treatment in Endometriosis-Associated Infertility Patients: a Retrospective Study. Am. J. Transl Res. 13 (4), 2399-2409.

Taylor, H. S., Kotlyar, A. M., and Flores, V. A. (2021). Endometriosis Is a Chronic Systemic Disease: Clinical Challenges and Novel Innovations. Lancet 397 (10276), 839-852. doi:10.1016/S0140-6736(21)00389-5

Wu, Y. B., Ni, Z. Y., Shi, Q. W., Dong, M., Kiyota, H., Gu, Y. C., et al. (2012). Constituents from Salvia Species and Their Biological Activities. Chem. Rev. 112 (11), 5967-6026. doi:10.1021/cr200058f

Wu, Y. H. (2016).Research of Reinforcing Kidney and Eliminating Blood Stasis to Prevent and Treat Side Effects in Induced by GnRH-A for Endometriosis with Kidney Deficiency and Blood Stasis after Surgery. Fuzhou: Fujian University of Traditional Chinese Medicine, 2-8. M Sc. Thesis

Zhang, J. R., and Zhao, Y. (2017). Effect of Huoxue Xiaoyi Recipe Combined with GnRH-A on Postoperative Recurrence of Endometriosis of Qi Stagnation and Blood Stasis. Asia Pac. Traditional Med. 13 (14), 148-149.

Zhang, L. Y., and Wang, X. H. (2019). Clinical Efficacy of the Bushen Quyu Therapy Plus GnRH-A on Endometriosis after Laparoscopic Surgery. Clin. J. Chin. Med. 11 (26), 115-117.

Zhang, Y. (2020). Clinical observation of dan'e fukang soft extracts combined with triptorelin acetate after laparoscopic surgery for endometriosis. J. Pract. Traditional Chin. Med. 36 (12), 1575-1576. Available at: https://kns.cnki.net.

Zhou, Z. H., Weng, Q., Zhou, J. H., and Zhou, J. (2012). Extracts of Salvia Miltiorrhiza Bunge on the Cytokines of Rat Endometriosis Models. Afr. J. Tradit Complement. Altern. Med. 9 (3), 303-314. doi:10.4314/ajtcam.v9i3.2 Zondervan, K. T., Becker, C. M., and Missmer, S. A. (2020). Endometriosis. N. Engl. J. Med. 382 (13), 1244-1256. doi:10.1056/NEJMra1810764

Conflict of Interest: The authors declare that the research was conducted in the absence of any commercial or financial relationships that could be construed as a potential conflict of interest.

Publisher's Note: All claims expressed in this article are solely those of the authors and do not necessarily represent those of their affiliated organizations, or those of the publisher, the editors and the reviewers. Any product that may be evaluated in this article, or claim that may be made by its manufacturer, is not guaranteed or endorsed by the publisher.

Copyright (ङ 2022 Gao, Shen, Jiang, Luan, Lin, Meng, Wang and Cong. This is an open-access article distributed under the terms of the Creative Commons Attribution License (CC BY). The use, distribution or reproduction in other forums is permitted, provided the original author(s) and the copyright owner(s) are credited and that the original publication in this journal is cited, in accordance with accepted academic practice. No use, distribution or reproduction is permitted which does not comply with these terms. 